\title{
Neuroligin: the synaptic multitasker
}

It is already known that the postsynaptic adhesion molecule neuroligin 1 , in complex with its axonal receptor, $\beta$-neurexin, is involved in presynaptic terminal formation. Now, new research published in Science shows that the same complex is also involved in postsynaptic differentiation, and that neuroligins have an important role in maintaining the functional balance of excitatory and inhibitory synapses.

Chih and co-workers first showed that neuroligin overexpression in cultured rat hippocampal cells increased both the formation of dendritic spines and the recruitment of postsynaptic molecules, including NMDA ( $N$-methyl-D-aspartate) receptors.

They then used two neuroligin 1 mutants to investigate the involvement of neuroligin in postsynaptic density molecule recruitment and scaffolding assembly. A dominant-negative mutant with a disrupted $\beta$-neurexin-binding site induced postsynaptic clustering of the scaffolding molecule postsynaptic density protein 95 (PSD-95), but the clusters were misaligned with the presynaptic terminals.

In the second mutant, the loss of neuroligin's PDZ-binding motif resulted in a predictable absence of PSD-95 clustering, but no severe effect on NMDA receptor clustering was seen, indicating that NMDA receptor recruitment is largely independent of PSD-95 clustering. Therefore, the PDZ-binding motif is required for PSD-95 clustering, whereas the $\beta$-neurexin-binding site is needed for the clusters to align with presynaptic terminals.

In rodents, there are three neuroligin isoforms, and knockdown of each single isoform resulted in a significant reduction in the number of excitatory and inhibitory terminals. This indicates that there is some functional overlap between the neuroligin isoforms.
Chih et al. also found that excitatory and inhibitory neurotransmission were not equally perturbed by neuroligin downregulation - inhibitory neurotransmission was affected far more than excitatory neurotransmission, which resulted in an imbalance of cellular activity. So far, the reasons for this preferential effect on inhibitory transmission are unknown, but the authors suggest that excitatory synapses might be better able to produce a compensatory upregulation in activity. Moreover, at normal neuroligin levels, a significant proportion of excitatory synapses are silent, and loss of these synapses will not alter cellular excitation.

Sarah Archibald

\section{(4) References and links} ORIGINAL REFERENCE PAPER Chih, B. et al. Control of excitatory and inhibitory synapse formation by neuroligins. Science 27 January 2005

(doi:10.1126/science.1107470)

FURTHER READING

Dean, C. et al. Neurexin mediates the assembly of presynaptic terminals. Nature Neurosci. 6, 708-716 (2003)

\section{Reach out and touch}

In the densely packed neocortex, neurons form synaptic connections with only a selected subset of their neighbours. Kalisman et al. investigated how neurons control this selective connectivity, and found that the axons of pyramidal neurons are not selective in terms of physical contact with neighbouring dendrites; rather, they touch the dendrites of all neighbouring neurons, but make synaptic contacts with only a few of them.

Although it is clear that pyramidal cell axons must select their targets at some level, it has been hard to discover whether they project to specific dendrites within the local microcircuit, or instead project 'randomly' and form synapses at only a subset of contact points. To distinguish between these possibilities, Kalisman and colleagues used simultaneous multineuron recording combined with confocal microscopy to compare the functional connections between cortical neurons with their structural properties.

The key finding of the confocal microscopy was that a pyramidal neuron makes physical contact with every other neighbouring pyramidal neuron in the local microcircuit. In fact, in most cases neurons 'touch' each other several times, and the likelihood that two neurons touch is unrelated to whether they are functionally connected (as revealed by simultaneous recordings). However, neurons that were functionally connected were more likely to be connected by putative synapses, as would be expected.

The authors interpret the apparently random formation of physical contact between neurons as a kind of tabula rasa - a flexible pattern of potential circuitry on which can be imposed any required functional microcircuit. The large number of contacts between neighbouring pyramidal neurons could also be important for cortical plasticity, as it might allow the formation of new synapses without axons or dendrites having to change their morphology.

Rachel Jones

\section{(2) References and links}

ORIGINAL RESEARCH PAPER Kalisman, $\mathrm{N}$. et al. The

neocortical microcircuit as a tabula rasa. Proc. Nat/Acad. Sci. USA 102, 880-885 (2005)

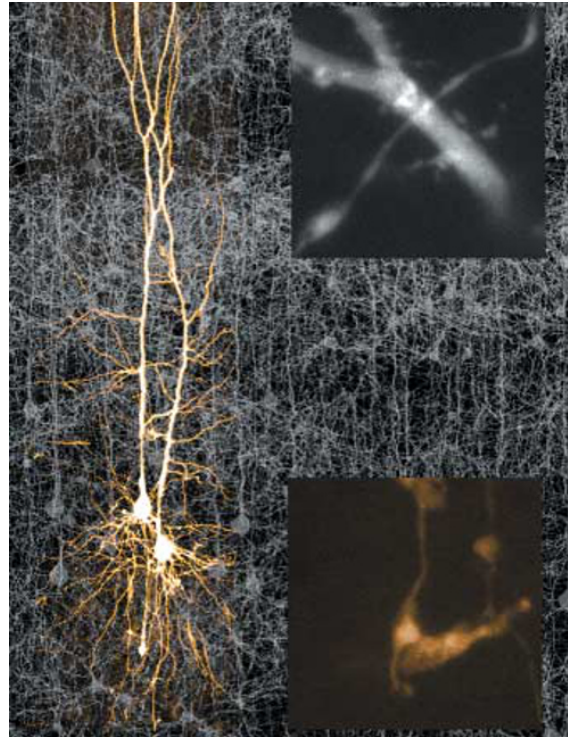

A confocal image of two layer 5 pyramidal cells on a background of reconstructed neurons, illustrating the fraction of choices for target cells. The upper insert shows an en passant axon 'touching' a dendrite without forming a bouton and the lower insert shows an en passant axon touching and forming a bouton at the touch site. The latter pair of neurons were synaptically connected, whereas the former were not. Image courtesy of H. Markram, Brain Mind Institute, Lausanne, Switzerland. 